ÉGYPTE monde arabe

\section{Égypte/Monde arabe}

7| 1991

Perceptions de la centralité de l'Égypte 1

\title{
Youssef Idris : Le roi du coton
}

Ibdâ' n9, septembre 1991

\section{Hana' 'Abd el-Fattah}

Traducteur : Mouna Akouri

\section{CpenEdition}

\section{Journals}

Édition électronique

URL : https://journals.openedition.org/ema/1188

DOI : 10.4000/ema. 1188

ISSN : 2090-7273

\section{Éditeur}

CEDEJ - Centre d'études et de documentation économiques juridiques et sociales

\section{Édition imprimée}

Date de publication : 30 septembre 1991

Pagination : 175-177

ISSN : 1110-5097

\section{Référence électronique}

Hana' 'Abd el-Fattah, «Youssef Idris : Le roi du coton », Égypte/Monde arabe [En ligne], 7| 1991, mis en ligne le 08 juillet 2008, consulté le 07 juillet 2022. URL : http://journals.openedition.org/ema/1188 ; DOI : https://doi.org/10.4000/ema. 1188

Ce document a été généré automatiquement le 7 juillet 2022.

Tous droits réservés 


\title{
Youssef Idris : Le roi du coton
}

\author{
Ibdâ' n 9, septembre 1991
}

\author{
Hana' 'Abd el-Fattah
}

Traduction : Mouna Akouri

\section{NOTE DE L'ÉDITEUR}

L'expérience avait réussi à soulever un débat sur l'idée de construire un théâtre et un amphithéâtre selon une architecture adaptée. Le ministre de la Culture de l'époque, $\mathrm{M}$. Tharwat 'Ukâcha décida que des ingénieurs-architectes bâtiraient un théâtre spécial dans le village d'al-May, proche de Danchawây, dans la province d'al-Minufiyya. Les ingénieurs s'inspirèrent de l'architecture du village pour ériger un théâtre à ciel ouvert réservé à la Troupe de théatre paysan de Danchawây et aux expériences similaires.

Il est des expériences théâtrales qui représentent un jalon essentiel dans la carrière d'un artiste. Malik al-Qutn ( Le roi du coton») de Youssef Idris, a été de celles-là, et ce fut même mon expérience théâtrale la plus importante. Monter cette pièce ne relevait pas tant pour moi du travail traditionnel de metteur en scène que d'une investigation du texte de Youssef Idris à travers les personnages de Qamhâwî Abû Ibrahîm, Al Sunbâtî Effendî, Muhammad, Umm Muhammad et d'autres... C'était la première fois qu'existait une relation intime et en même temps dialectique entre les acteurs et les personnages. Le mot «intimité » est à prendre ici dans le sens de "continuité vivante » entre les interprètes et leurs rôles, les personnages qui figuraient des paysans et les paysans véritables, ceux-là n'ayant pas besoin de jouer un rôle pour incarner les premiers. Quant au rapport dialectique, il est né de la nature de cette structure dramatique nouvelle. Pour moi, metteur en scène, il ne s'agissait pas seulement de faire appel à des acteurs/paysans pour qu'ils viennent jouer leurs préoccupations quotidiennes ou leurs aspirations; en confrontant le texte au quotidien réel de ces paysans, il s'agissait aussi de mettre le premier à l'épreuve, d'en analyser les significations, d'en tester l'atmosphère... Je pense que cette mise à l'épreuve artistique imposée au «Roi du 
Coton ", en questionnant l'authenticité de la peinture des moeurs dans un village égyptien ou des personnages mis en scène, fut unique en son genre.

2 Cette expérience devait donner naissance à un élément nouveau du fait qu'elle ne constituait ni une interprétation fidèle à la lettre du texte ni un débordement des limites de son contenu; elle fit tout simplement apparaître une réalité nouvelle, originale : elle posait, d'une part, le principe de la créativité collective d'une troupe de théâtre, et d'autre part, soulevait la question à laquelle metteurs en scène et hommes de théatre cherchent toujours une réponse : de quelle marge de liberté un metteur en scène dispose-t-il lorsqu'il aborde une oeuvre dramatique selon son interprétation propre, donc singulière? La pièce Malik al-Qutn, présentée avec les paysans de Danchawây, répondait concrètement à cette question. En lisant le texte, je prêtais l'oreille aux objections des acteurs/paysans ; tantôt je sentais leur approbation, tantôt leur désapprobation quant à certains passages de la pièce concernant leur vie, leur langage. Lorsqu'ils n'étaient pas d'accord, ils insistaient pour introduire des modifications dans le texte. Mais, outre que ces modifications ne constituaient pas un but en soi, elles ne s'arrêtaient pas non plus aux seuls éléments formels relatifs au lieu, au temps, au dialogue. Les transformations souhaitées dépassaient ce cadre et proposaient une réponse nouvelle à la question récurrente sur les contraintes auxquelles doit se plier le metteur en scène et sur son droit à introduire des changements dans la structure même de l'écrit dramatique.

3 Le changement, ce n'est pas moi qui l'ai voulu... Mais un jour, 'Uthmân al-Khabayrî qui interprétait le personnage de Qamhâwî Abu Ibrâhîm - s'est brusquement interrompu et a refusé de jouer; je ne l'avais pas entendu protester clairement ni à voix haute; tout simplement, il s'était mis à modifier son jeu à sa convenance. Il s'est accroupi de la manière dont il pensait que devait le faire Qamhâwi Abû Ibrâhîm - le paysan salarié - devant al-Sunbâtî Effendî - propriétaire de la terre et de ceux qui y travaillent. 'Uthmân al-Khabayrî m'a appris comment on s'asseyait par terre ; il tenait alors sa tige de roseau avec laquelle il traçait minutieusement des repères sur le sable, l'index de la main gauche pointé vers le front en signe de profonde réflexion. Quant à 'Abd al Wârith - qui dans la vie était courtier en veaux et jouait le rôle d'al-Sunbâtî Effendî - il m'a expliqué la pertinence d'être assis d'un côté plutôt que de l'autre selon le contexte dramatique... D'autre part, les acteurs-paysans trouvaient le dialogue, du moins dans certaines parties, peu représentatif de leur manière de parler. C'est ce qui les détermina également à introduire les changements qu'ils jugeaient nécessaires et conformes à ce qu'ils voyaient et ressentaient. Le texte dramatique devint dès lors une sorte de pâte malléable entre les mains des acteurs, qui le modelaient à leur idée et selon la nature de la situation vécue. Ils ont cependant conservé l'armature générale du texte et n'ont modifié ni l'idée de Youssef Idris ni son message particulier; ils ont seulement cherché à le reformuler en lui insufflant l'atmosphère du village de Danchawây de manière à lui faire exprimer les problèmes de leur village et leurs propres préoccupations lorsqu'ils ont été convaincus que ces personnages les représentaient. C'est donc toujours en partant de ce principe que l'on a modifié certaines situations, que de nouvelles scènes ont été greffées sans pour autant porter atteinte à l'essence du texte original ; celui-ci avait ainsi acquis une touche de sincérité et d'authenticité. La pièce n'était plus une simple représentation jouée devant un public de paysans dans l'espace du village réservé à cet effet; elle avait pris l'allure d'une célébration rituelle. Le but n'était donc pas d'empiéter sur le domaine de 
l'écrivain, ni d'enfreindre son système ou son organisation du texte, mais de tenter de le réinterpréter.

4 La représentation dramatique du "Roi du Coton » devenait donc en elle-même un stimulant pour l'étude; étude de la nature du lieu, du temps réel de l'oeuvre, des hommes qu'on y peignait. L'expérience dans sa totalité fut une renaissance pour Youssef Idris, il renaissait artistiquement dans l'esprit des paysans sur lesquels il avait écrit. Ces derniers ont été en même temps acteurs, interprètes, expérimentateurs et spectateurs d'une expérience vécue ; ils ont prouvé - inconsciemment - que le texte dramatique n'était en définitive qu'un élément esthétique parmi ceux qui constituent la représentation dans son ensemble.

5 «La Troupe de théâtre paysan » du village de Danchawây a joué plus de cent fois «Le Roi du Coton» dans diverses régions d'Egypte, villages et villes. Une des représentations fut donnée dans les années 70 à l'ouverture du premier festival d'art dramatique des troupes de province qui se déroulait sur les planches du Théatre national, ces mêmes planches qui l'avaient vue jouer à la fin des années $50 . .$. Youssef Idris avait eu connaissance de notre travail. À la fin de la représentation, il se leva et alla embrasser un à un les acteurs dé la troupe puis il dit avec gravité: "Je n'aurais jamais rêvé pouvoir écrire avec autant de sincérité... Vous avez réussi mieux que moi à matérialiser ce que je rêvais d'écrire... et vous l'avez fait avec simplicité et profondeur dans votre Roi du Coton. »

INDEX

Mots-clés : Idris (Youssef), littérature 